\title{
Simultaneous measurement of displacement and temperature based on two cascaded balloon-like bent fibre structures
}

\author{
Ke Tian \\ Harbin Engineering University \\ Ruoning Wang \\ Harbin Engineering University \\ Meng Zhang \\ Harbin Engineering University
}

See next page for additional authors

Follow this and additional works at: https://arrow.tudublin.ie/prcart

Part of the Electrical and Computer Engineering Commons, and the Optics Commons

\begin{abstract}
Recommended Citation
Ke Tian, Ruoning Wang, Meng Zhang, Xianfan Wang, Xin Wang, Guoyong Jin, Elfed Lewis, Gerald Farrell, Pengfei Wang, Simultaneous measurement of displacement and temperature based on two cascaded balloon-like bent fibre structures, Optical Fiber Technology, Volume 58, 2020, 102277, ISSN 1068-5200, DOI: 10.1016/j.yofte.2020.102277.
\end{abstract}

This Article is brought to you for free and open access by the Photonics Research Centre at ARROW@TU Dublin. It has been accepted for inclusion in Articles by an authorized administrator of ARROW@TU Dublin. For more information, please contact arrow.admin@tudublin.ie, aisling.coyne@tudublin.ie,gerard.connolly@tudublin.ie. Funder: National Natural Science Foundation of China (NSFC); Science Foundation Ireland

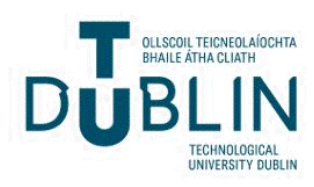




\section{Authors}

Ke Tian, Ruoning Wang, Meng Zhang, Xiafan Wang, Xin Wang, Guoyong Jin, Elfed Lewis, Gerald Farrell, and Pengfei Wang

This article is available at ARROW@TU Dublin: https://arrow.tudublin.ie/prcart/15 


\title{
Simultaneous measurement of displacement and temperature based on two cascaded balloon-like bent fibre structures
}

\author{
Ke Tian ${ }^{\mathrm{a}, \mathrm{b}}$, Ruoning Wang ${ }^{\mathrm{a}}$, Meng Zhang ${ }^{\mathrm{a}}$, Xianfan Wang ${ }^{\mathrm{c}}$, Xin Wang ${ }^{\mathrm{a}}$, Guoyong Jin ${ }^{\mathrm{b}}$, \\ Elfed Lewis $^{\mathrm{d}}$, Gerald Farrell ${ }^{\mathrm{e}}$, Pengfei Wang ${ }^{\mathrm{a}, \mathrm{f}, *}$ \\ ${ }^{a}$ Key Laboratory of In-fiber Integrated Optics of Ministry of Education, College of Science, Harbin Engineering University, Harbin 150001, China \\ ${ }^{\mathrm{b}}$ College of Power and Energy Engineering, Harbin Engineering University, Harbin 150001, China \\ ${ }^{\text {c }}$ State Key Laboratory of Modern Optical Instrumentation, Zhejiang University, Hangzhou 310027, China \\ ${ }^{\mathrm{d}}$ Optical Fibre Sensors Research Centre, Department of Electronic and Computer Engineering, University of Limerick, Limerick, Ireland \\ ${ }^{\mathrm{e}}$ Photonics Research Centre, Technological University Dublin, Kevin Street, Dublin 8, Ireland \\ ${ }^{\mathrm{f}}$ Key Laboratory of Optoelectronic Devices and Systems of Ministry of Education and Guangdong Province, College of Optoelectronic Engineering, Shenzhen University, \\ Shenzhen 518060, China
}

\section{A R T I C L E I N F O}

\section{Keywords:}

Modal interferometer

Simultaneous measurement

Displacement measurement

Temperature measurement

\begin{abstract}
A B S T R A C T
A low-cost optical fibre sensor based on two cascaded balloon-like bent fibre (BBF) structures for simultaneous displacement and temperature measurement is reported. The sensor is fabricated by cascading two balloon-like bent single-mode fibres (SMFs) which with different bending radii, generating two separate interference dips within a limited wavelength range. The wavelength of the two interference dips exhibits different responses to external displacement and temperature variations, hence simultaneous measurement of displacement and temperature is realized. Experimental results show that the proposed optical fibre sensor achieves a displacement sensitivity of $-318.8 \mathrm{pm} / \mu \mathrm{m}$ and a temperature sensitivity of $47.4 \mathrm{pm} /{ }^{\circ} \mathrm{C}$. Taking advantage of its lowcost, ease of fabrication, and experimentally determined high sensitivity, the sensor in this investigation can be potentially applied in both displacement and temperature measurement fields.
\end{abstract}

\section{Introduction}

Monitoring of displacement is a key requirement in many measurement application fields, such as microimaging, robotics, astronautics, and structural health monitoring (SHM) [1,2]. In order to meet a variety of different measurement demands, various types of displacement sensors have been reported in recent years. Among them, optical fibre displacement sensors have regularly gained an edge over conventional electronic sensors due to their particular advantages including light weight, compact size, electromagnetic immunity and remote sensing capability. To date, several different fibre configurations have been adopted to realize displacement measurement, including fibre grating structures e.g. fibre Bragg gratings (FBGs) [3,4], tilted FBGs [5], long-period gratings (LPGs) [6], and various fibre interferometers including singlemode-multimode-singlemode (SMS) structures $[7,8]$, Mach-Zehnder interferometers (MZIs) $[9,10]$, a Sagnac interferometer [11], and a Fabry-Perot interferometer (FPI) [12]. Compared with fibre-grating-based displacement sensors, fibre-interferometer-based displacement sensors normally provide higher measurement accuracy and a wider measurement range. However, in the case of many fibre interferometer devices, the complicated fabrication process and fragile structure may hinder their widespread application in industrial or commercialized optical fibre displacement sensing.

Recently, an easy-to-fabricate, structurally robust balloon-like interferometer has been reported and has attracted a great deal of research attention. However, this structure has proven to have a relatively high temperature dependence of up to $418 \mathrm{pm} /{ }^{\circ} \mathrm{C}$ [13], and in the absence of a stripped coating process, its temperature sensitivity can reach $-2465 \mathrm{pm} /{ }^{\circ} \mathrm{C}[14]$. This means that an undesirable cross-sensitivity induced by temperature is inevitable in the application of such a structure for measurement of other parameters. In order to eliminate temperature induced cross-sensitivity, simultaneous multi-parameter measurement is the most commonly used method [15]. Based on previous research, simultaneous measurement usually requires the incorporation of two separate measurement elements to introduce active sensing and reference (temperature based) interference patterns. Several fibre configurations are frequently chosen to realize the goal of

\footnotetext{
* Corresponding author.

E-mail addresses: ketian@hrbeu.edu.cn (K. Tian), pengfei.wang@tudublin.ie (P. Wang).
} 
simultaneous measurement, such as FBGs [16-19], LPGs [20,21], polarization maintaining fibre (PMF) [22], and photonics crystal fibre (PCF) $[23,24]$. However, combining these fibre configurations further increases the complexity and cost of the overall sensor system. Therefore, there is a clear need to develop a simple and cost-effective optical fibre sensor structure for simultaneous measurement of displacement and temperature.

In this article, a low-cost optical fibre sensor based on two cascaded balloon-like bent fibre (BBF) structures for simultaneous measurement of displacement and temperature is experimentally demonstrated. The sensor is fabricated by simply bending a standard SMF into a balloon shape with the help of a capillary tube, without the requirement for any expensive equipment. Through cascading two balloon-like bent SMFs with different bending radii, two separate interference dips can be obtained within the wavelength domain of the detector and used for simultaneous measurement of displacement and temperature. Benefiting from its advantages of low-cost, ease of fabrication and high measurement sensitivity, the sensor of this investigation may therefore find use in applications in accurate displacement and temperature measurement fields.

\section{Principle and fabrication}

The schematic diagram of the cascaded balloon-like bent SMF structures is illustrated in Fig. 1. The sensor consists of two balloon-like bent SMFs, but with different bending radii of $r_{1}$ and $r_{2}$, and different lengths of $L_{1}$ and $L_{2}$. As depicted in Fig. 1, the light from an optical source initially propagates along the core of the input SMF in fundamental mode. When the light arrives at the first balloon-like section, owing to the bend of the waveguide, a portion of the light from the SMF core is coupled into the cladding and excites the cladding modes. These modes propagate within the SMF cladding, and after passing through the bend region, part of them are recoupled back to the SMF core. Interference therefore occurs because of the effective RI differences of the core and cladding modes and the optical path difference (OPD) experienced by the light signals. When the light arrives at the second balloon-like bent section, the above-mentioned interference occurs again. In the case of a two-mode modal interferometer, the output intensity can be expressed as [25]

$I=I_{c o}+I_{c l}+2 \sqrt{I_{c o} I_{c l}} \cos \left(\frac{2 \pi}{\lambda} L_{e f f} \Delta n_{e f f}\right)$

where $I_{c o}$ and $I_{c l}$ represent the intensity of the core mode and cladding modes, $\lambda$ is the free space wavelength, $L_{e f f}$ is the effective interference length and $\Delta n_{\text {eff }}=n_{c o}-n_{c l}$ denotes the effective RI difference between the core and cladding modes. When the phase difference meets an odd multiple of $\pi$, the center wavelength of the interference dip can be expressed as [26]

$\lambda_{s}=\frac{2}{(2 s+1)} L_{e f f} \Delta n_{e f f}$

where $s$ is an integer $(s=1,2,3 \ldots)$. The free spectral range (FSR) of the

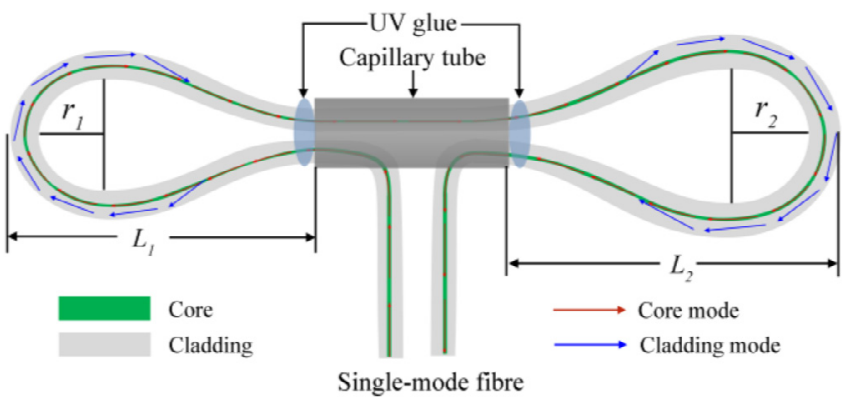

Fig. 1. Schematic diagram of the cascaded BBF structures. transmission spectrum can be calculated from Eq. (2) as

$F S R=\frac{\lambda_{s}^{2}}{L_{e f f} \Delta n_{\text {eff }}}$

Since the two BBFs have different bending radii, their effective interference length of $L_{e f f}$ and the effective RI difference $\left(\Delta n_{e f f}\right)$ are different, resulting in different resonance wavelengths according to Eq. (2). When the external displacement or temperature change, the value of $L_{\text {eff }}$ and $\Delta n_{\text {eff }}$ also change which leads to a shift in the resonance wavelengths. Therefore, in principle the BBF structure can be used for simultaneous measurement of displacement and temperature.

Additionally, due to the existence of the elasto-optical effect, the bend also effects the physical RI distribution inside the SMF. For a bent SMF, its originally symmetric radial RI distribution becomes asymmetrical, and the redistributed RI status can be equivalent to a distribution superimposed on that of a straight SMF, which can be expressed as

$n_{1}=n_{0}\left(1+\frac{x}{r_{e f f}}\right)$

where $n_{0}$ is the RI of the straight fibre, $x$ is the displacement in the normal direction to the bent fibre axis, and $r_{\text {eff }}$ is the equivalent bending radius which can be calculated as follows [27]:

$r_{e f f}=\frac{r}{1-n_{0}^{2} / 2\left[P_{12}-v\left(P_{11}+P_{12}\right)\right]}$

where $r$ is the bending radius of the fibre, $P_{11}$ and $P_{12}$ are components of the photoelastic tensor, and $v$ is the Poisson ratio.

Numerical simulations based on the Beam Propagation Method (BPM) have been carried out to investigate the optical field distribution within the bent fibre. According to Eq. (4), the RI distribution of the bent fibre can be established in a straight fibre model. To simplify the simulation process, only the section with the largest curvature (the top of the balloon-like structure) was considered because this section has a uniform bending radius. The simulation geometry/RI parameters were set as: the diameters and RIs for the SMF core/cladding are 8.3/125 $\mu \mathrm{m}$ and 1.4504/1.4447, respectively, and the simulation length of the SMF is $1 \mathrm{~cm}$. When the bending radii were set to $10 \mathrm{~mm}, 8 \mathrm{~mm}, 6 \mathrm{~mm}$ and $4 \mathrm{~mm}$, for an incident wavelength of $1550 \mathrm{~nm}$, the simulation results are shown in Fig. 2. It can be observed from Fig. 2 that optical field is mainly concentrated on the outside of the bent fibre. With a decrease in the bending radius from $10 \mathrm{~mm}$ to $4 \mathrm{~mm}$, the optical field intensity of the cladding modes excited in the SMF cladding layer increases. The optical field intensity distribution of the core and cladding mode due to modal interference results in a difference in the extinction ratio (ER) of the interference dips. Therefore, a suitable range of bending radii needs to be determined experimentally to obtain an effective interference pattern.

Fig. 3 shows the measured transmission spectra in the range of

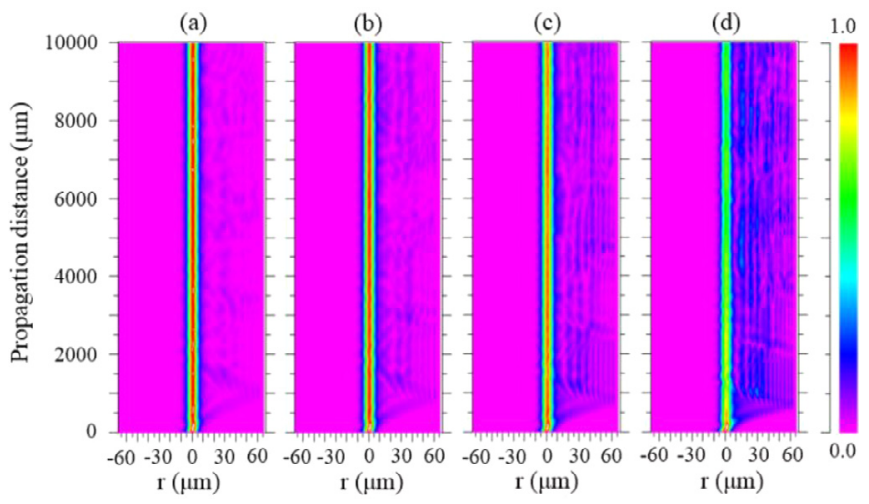

Fig. 2. Simulated optical field distribution of the BBF structure with different bending radii (a) $r=10 \mathrm{~mm}$; (b) $r=8 \mathrm{~mm}$; (c) $r=6 \mathrm{~mm}$; (d) $r=4 \mathrm{~mm}$. 


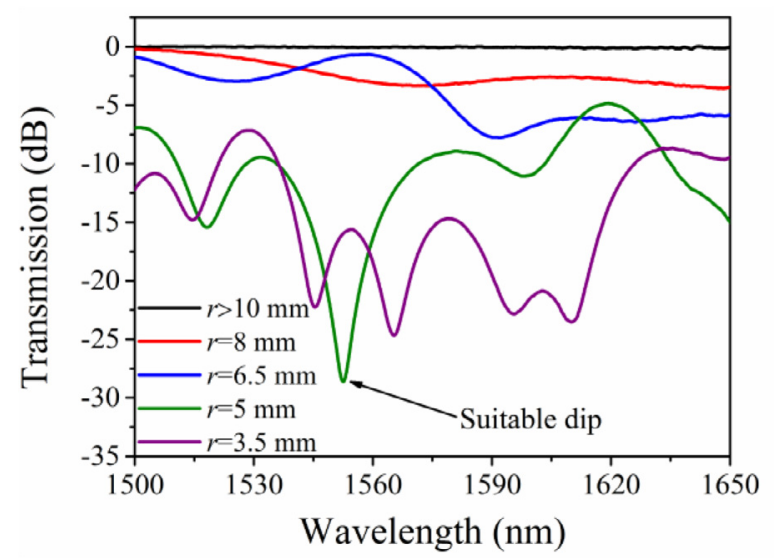

Fig. 3. Measured transmission spectra of the BBF structure with different bending radii.

$1500-1650 \mathrm{~nm}$ of a BBF structure with different bending radii. It can be seen that when the bending radius is greater than $10 \mathrm{~mm}$, no clear interference dips were observed since very little light propagating in the SMF core is coupled into the cladding. As the bending radius decreases, more light is coupled into the cladding, resulting in an increase of transmission loss. Interference patterns appear when the bending radius was $8 \mathrm{~mm}$ and $6.5 \mathrm{~mm}$, and the resulting interference dips do not have a good ER. When the bending radius was further decreased to $5 \mathrm{~mm}$, an interference dip with enhanced ER (more than $18 \mathrm{~dB}$ ) occurred at $1552 \mathrm{~nm}$. With further decreases in the bending radius to $3.5 \mathrm{~mm}$, more interference dips were detected, but the induced interference dips possess a smaller ER compared with the $5 \mathrm{~mm}$ bending case. If the bending radius continues to decease below $2.5 \mathrm{~mm}$, the SMF is more susceptible to fracture, and the light signal in this case is weak because excess light is not only coupled into the cladding, but also out the cladding, resulting in large power loss. Therefore, based on the initial results obtained in this experiment, an appropriate bending radius for balloon-like structure to form an effective interference pattern should be in the region of $5 \mathrm{~mm}$.

To fabricate the cascaded BBF structures, a length of standard SMF (SMF-28, Corning) was initially stripped of its polymer coating over an appropriate length. Next, both free ends of the SMF were inserted into a Teflon capillary tube for which the length and inner diameter are $1.5 \mathrm{~cm}$ and $800 \mu \mathrm{m}$, respectively. By moving this capillary tube along the fibre, the bending radius of the balloon-like structure can be flexibly adjusted. Based on this method, two balloon-like structures with different bending radii were obtained, and the entire structure was finally immobilized using ultraviolet (UV) glue.

The bending radius and the length of the two balloon-like structures was measured. The smaller balloon-like structure (BBF1) has an $r_{1}$ of $4.96 \mathrm{~mm}$ and $L_{1}$ of $17.58 \mathrm{~mm}$, the larger balloon-like structure (BBF2) has an $r_{2}$ of $5.37 \mathrm{~mm}$ and $L_{2}$ of $19.18 \mathrm{~mm}$. The transmission spectra of the individual BBF1, BBF2, and the cascaded structure were measured and are shown in Fig. 4. It can be seen that two interference dips with high ER values were detected in the cascaded structure over the wavelength range of 1500-1650 $\mathrm{nm}$. The central wavelength of dip 1 and dip 2 were located at $1550.8 \mathrm{~nm}$ and $1605.8 \mathrm{~nm}$, respectively. Benefiting the high interference visibility of dip 1 and dip 2, these two interference dips formed by BBF1 and BBF2 were chosen for the measurement of this investigation.

Based on the above discussions, when the external displacement and temperature change, the resulting wavelength shift of dip 1 and dip 2 can be expressed as follows:

$\Delta \lambda_{\text {dip } 1}=K_{D 1} \cdot \Delta D+K_{T 1} \cdot \Delta T$

$\Delta \lambda_{\operatorname{dip} 2}=K_{D 2} \cdot \Delta D+K_{T 2} \cdot \Delta T$

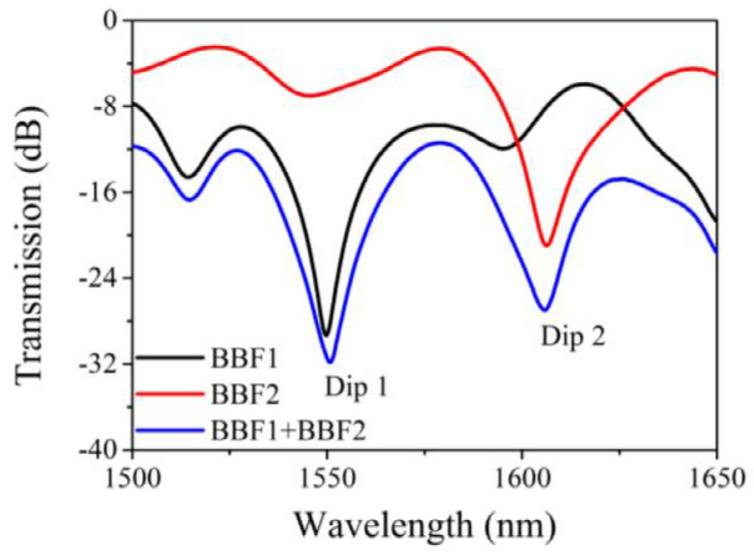

Fig. 4. Transmission spectra of the BBF1, BBF2 and the cascaded fibre structures.

where $\Delta D$ and $\Delta T$ denote the variation of displacement and temperature; $K_{D 1}, K_{D 2}, K_{T 1}$ and $K_{T 2}$ are the sensitivity coefficients of dip 1 and dip 2 in response to displacement and temperature, respectively. Once these coefficients are determined experimentally, and based on Eqs. (6) and (7), a demodulation matrix can be established to realize the goal of simultaneous measurement of displacement and temperature.

\section{Experimental results and discussions}

The experimental setup for micro-displacement test is illustrated in Fig. 5, where a supercontinuum light source (SCS, YSL SC-series, China) was used to provide broadband light and an optical spectrum analyzer (OSA, YOKOGAWA AQ6370D, Japan) with a spectral resolution of $20 \mathrm{pm}$ was used to monitor the spectral response of the sensor. The sensor sample was mounted horizontally and fixed on a lifting stage of adjustable height. A linear translation stage with a displacement resolution of $10 \mu \mathrm{m}$ was used to accurately change the displacement applied to the balloon-like section. The smaller balloon-like bent SMF was placed in contact with the translation stage and used for displacement measurement, thus while the larger radius balloon-like bent SMF was entirely free from external displacement change and therefore was subject only to temperature changes.

Fig. 6 shows the measured spectral response of the sensor when subjected to a $0-90 \mu \mathrm{m}$ displacement range at a constant temperature of $20{ }^{\circ} \mathrm{C}$. A complete measurement cycle was performed to test the hysteresis of the sensor for displacement measurement. In this case, the displacement was initially increased from 0 to $90 \mu \mathrm{m}$ and then decreased from $90 \mu \mathrm{m}$ back to 0 with a step of $10 \mu \mathrm{m}$. The corresponding spectral evolution results are shown in Fig. 6(a) and (b), respectively. From the results presented in Fig. 6(a), it is clear that the two separate interference dips exhibit different individual responses to external

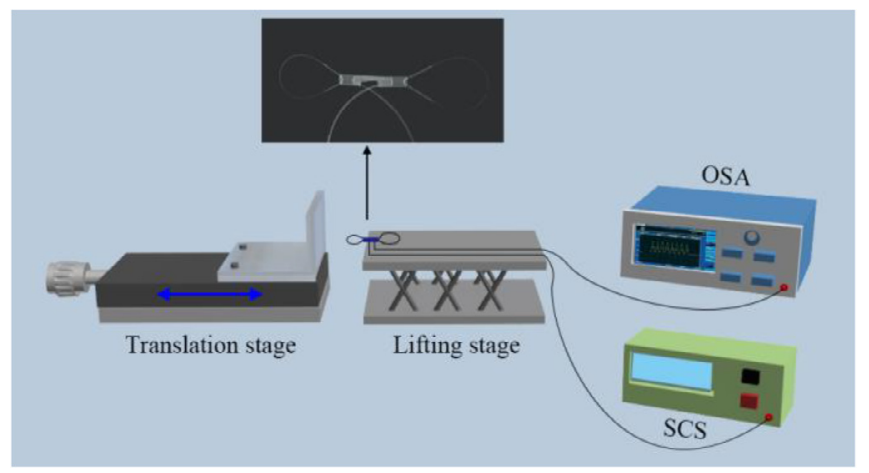

Fig. 5. Schematic diagram of the experimental setup for the displacement test. 

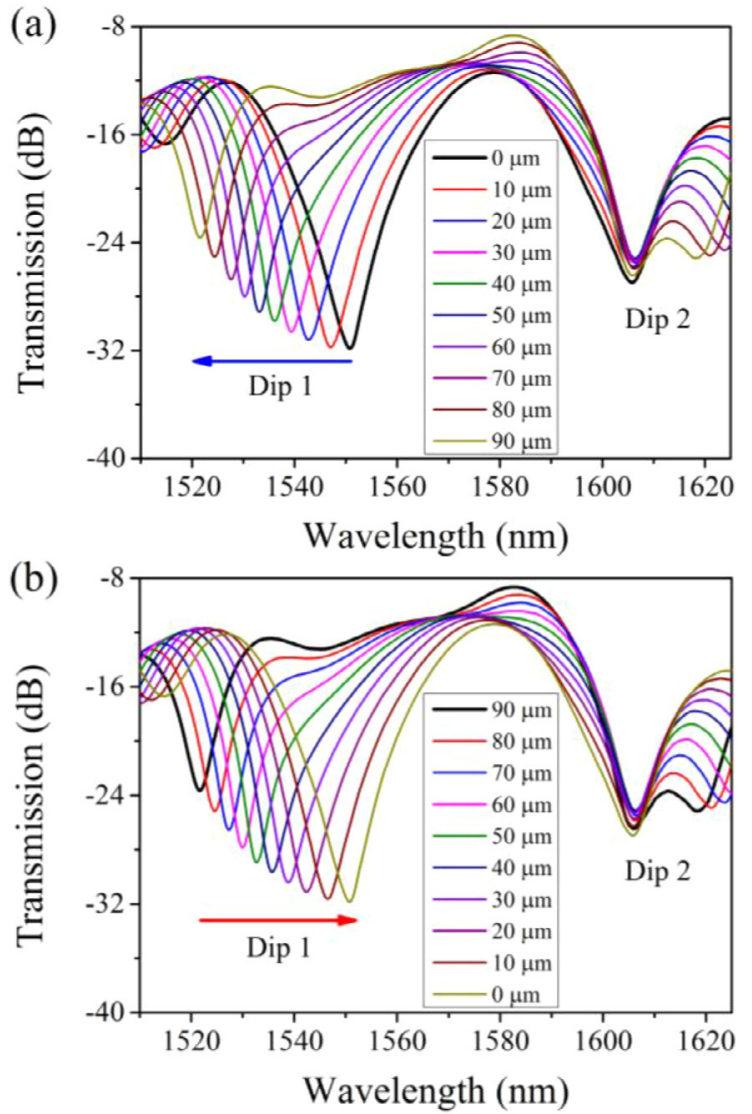

Fig. 6. Measured spectral responses at different displacement values (a) displacement increasing; (b) displacement decreasing.

displacement change. The smaller balloon-like bent SMF dip 1 exhibits a clear blue-shift in the wavelength domain, while the larger balloonlike bent SMF dip 2 shows no wavelength shift as the displacement was increased from 0 to $90 \mu \mathrm{m}$. For the second half of the cycle when the applied displacement was reduced, the evolution of dip 1 of Fig. 6(b) shows a clear red-shift and is therefore opposite to the displacement increasing case.

From the above spectral response results, it is clearly demonstrated that dip 2 is immune to external displacement change, which means its displacement sensitivity can be expressed as 0 . Conversely, external displacement variations strongly influence the location of the central wavelength of dip 1. Fig. 7 shows the wavelength of dip 1 as a function of displacement for the displacement increasing and decreasing cases. From the linear fitting results, the sensor exhibits an excellent linear

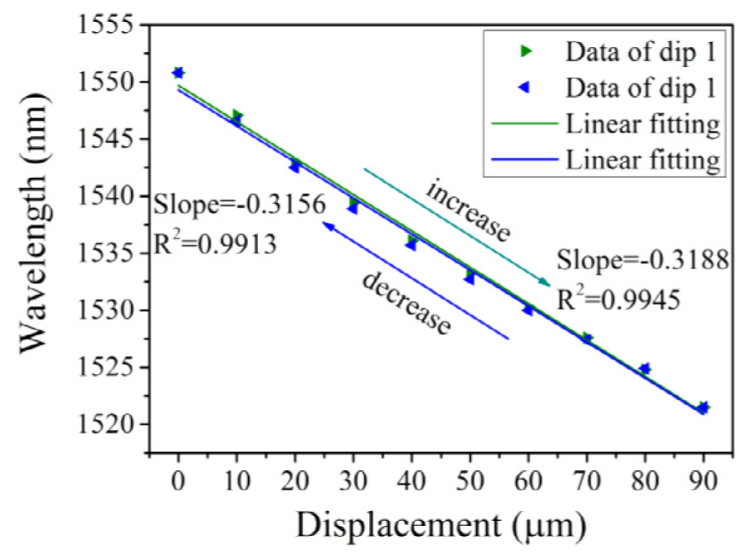

Fig. 7. Dip 1 wavelength versus displacement and the linear fitting.
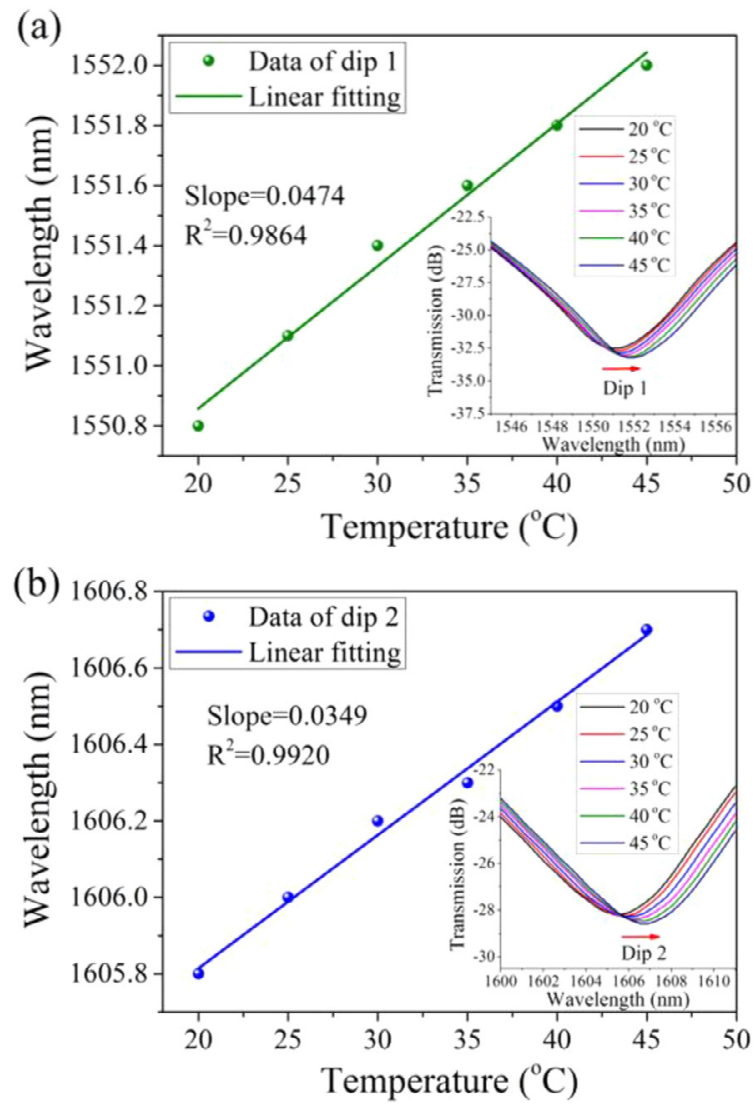

Fig. 8. Dip wavelength versus temperature and the linear fitting (a) result of dip 1 and its spectral evolution inset in the figure; (b) result of dip 2 and its spectral evolution inset in the figure.

response to external displacement variation, and the displacement sensitivities within the displacement range cases were calculated to be $-318.8 \mathrm{pm} / \mu \mathrm{m}$ (increasing) with an $\mathrm{R}^{2}$ of 0.9945 and $-315.6 \mathrm{pm} / \mu \mathrm{m}$ (decreasing) with an $\mathrm{R}^{2}$ of 0.9913 , respectively. Based on the observed spectral response characteristics and the calculated displacement sensitivities, it is also evident that there is no discernible hysteresis present for the displacement measurement.

To investigate the temperature response of the sensor, the sensor was placed in a temperature-controlled chamber (ESPEC SH-222, Japan), in which the inside temperature could be easily adjusted by altering the set value on an external operating panel. In this case, the temperature was increased from $20{ }^{\circ} \mathrm{C}$ to $45{ }^{\circ} \mathrm{C}$ with a step of $5{ }^{\circ} \mathrm{C}$, and the corresponding spectral response and data fitting results of dip 1 and dip 2 are shown in Fig. 8(a) and (b), respectively. It can be seen from the insets in Fig. 8(a) and (b) that both dip 1 and dip 2 exhibit a redshift in the wavelength domain as the temperature increases. The linear fitting results show these two dips exhibit a good linear response to temperature change, and the temperature sensitivities of dip 1 and dip 2 were determined to $47.4 \mathrm{pm} /{ }^{\circ} \mathrm{C}$ with $\mathrm{R}^{2}$ of 0.9864 and $34.9 \mathrm{pm} /{ }^{\circ} \mathrm{C}$ with $\mathrm{R}^{2}$ of 0.9920 , respectively.

The above experimental results clearly show that dip 1 and dip 2 have different response characteristics for displacement and temperature. The smaller BBF dip1 is sensitive to external displacement and temperature variation, while the larger BBF dip 2 is independent of displacement change which enables the combination of the two BBFs to be used for simultaneous measurement of displacement and temperature. Based on Eqs. (6) and (7) and the displacement and temperature sensitivity coefficients determined above, the demodulation matrix can be written as follows: 
Table 1

Comparison of the sensing performance of different sensors for displacement and temperature measurement.

\begin{tabular}{|c|c|c|c|}
\hline $\begin{array}{l}\text { Measurement } \\
\text { configuration }\end{array}$ & $\begin{array}{l}\text { Displacement } \\
\text { sensitivity (pm/ } \\
\mu \mathrm{m})\end{array}$ & $\begin{array}{l}\text { Temperature } \\
\text { sensitivity }(\mathrm{pm} / \\
\left.{ }^{\circ} \mathrm{C}\right)\end{array}$ & $\begin{array}{l}\text { Simultaneous } \\
\text { measurement }\end{array}$ \\
\hline FBG [3] & 0.0643 & 16.9 & Yes \\
\hline FBG [4] & 0.058 & - & No \\
\hline Tilted FBG [5] & $-0.0086\left(\mathrm{~m}^{-1}\right)$ & - & No \\
\hline LPG [6] & 220 & - & No \\
\hline MMI [7] & 35.5 & - & No \\
\hline MMI [8] & $0.51(\mathrm{~dB} / \mu \mathrm{m})$ & $-0.365\left(\mathrm{~dB} /{ }^{\circ} \mathrm{C}\right)$ & No \\
\hline MZI [9] & 835 & $46-85$ & No \\
\hline MZI [10] & $\begin{array}{l}-0.385(\mathrm{~dB} / \\
\mu \mathrm{m})\end{array}$ & $-0.036\left(\mathrm{~dB} /{ }^{\circ} \mathrm{C}\right)$ & No \\
\hline $\begin{array}{l}\text { Sagnac interferometer } \\
{[11]}\end{array}$ & - & - & No \\
\hline FPI $[12]$ & - & - & No \\
\hline $\begin{array}{l}\text { Bent fibre with LPG } \\
\text { [20] }\end{array}$ & -306 & 42.9 & Yes \\
\hline $\begin{array}{l}\text { Cascaded balloon-like } \\
\text { fibre structures } \\
\text { (this work) }\end{array}$ & -318.8 & 47.4 & Yes \\
\hline
\end{tabular}

$\left[\begin{array}{l}\Delta D \\ \Delta T\end{array}\right]=\left[\begin{array}{cc}-318.8 \mathrm{pm} / \mu \mathrm{m} & 47.4 \mathrm{pm} /{ }^{\circ} \mathrm{C} \\ 0 & 34.9 \mathrm{pm} /{ }^{\circ} \mathrm{C}\end{array}\right]^{-1}\left[\begin{array}{l}\Delta \lambda_{\text {Dip } 1} \\ \Delta \lambda_{\text {Dip } 2}\end{array}\right]$

Moreover, according to the OSA with a resolution of $20 \mathrm{pm}$ used in the experiments and assuming a suitable calibration, the displacement and temperature measurement resolution of the optical fibre sensor of this investigation were calculated to be $0.06 \mu \mathrm{m}$ and $0.42{ }^{\circ} \mathrm{C}$, respectively.

Table 1 includes a direct comparison of the displacement and temperature sensing performance between the sensor described in this investigation and the other sensors cited in this article. It can be seen that the cascaded balloon-like bent fibre structures has competitive displacement and temperature sensitivity compared to the previous cases. Moreover, the sensor of this investigation can achieve simultaneous measurement of displacement and temperature, which supports its potential applications in the field of displacement and temperature measurement.

\section{Conclusion}

In summary, a low-cost optical fibre sensor based on two cascaded balloon-like bent fibre structures for simultaneous displacement and temperature measurement has been presented. The sensor was simply fabricated by cascading two balloon-like bent SMFs to generate independent interference dips in the transmission spectrum. The smaller balloon-like bent SMF interference dip was sensitive to both displacement and temperature variation, while the larger balloon-like SMF was entirely strain relieved and hence introduced a reference dip to realize unambiguous displacement and temperature measurement. Experimental results show a displacement sensitivity of $-318.8 \mathrm{pm} / \mu \mathrm{m}$ over the displacement range of $0-90 \mu \mathrm{m}$ and a temperature sensitivity of $47.4 \mathrm{pm} /{ }^{\circ} \mathrm{C}$ over the temperature range of $20-45{ }^{\circ} \mathrm{C}$ were achieved. Such a low-cost optical fibre sensor is highly sensitive and is without hysteresis, which provides great application potential for use in many robust displacement and temperature measurement scenarios.

\section{CRediT authorship contribution statement}

Ke Tian: Conceptualization, Methodology, Validation, Investigation, Data curation, Writing - original draft. Ruoning Wang: Methodology, Investigation. Meng Zhang: Methodology, Investigation. Xianfan Wang: Software. Xin Wang: Visualization. Guoyong Jin: Writing - review \& editing. Elfed Lewis: Writing - review \& editing.
Gerald Farrell: Writing - review \& editing. Pengfei Wang: Writing review \& editing, Supervision.

\section{Declaration of Competing Interest}

The authors declare that they have no known competing financial interests or personal relationships that could have appeared to influence the work reported in this paper.

\section{Acknowledgments}

This work was supported by the National Natural Science Foundation of China (NSFC) (61935006), the Fundamental Research Funds for the Central Universities (HEUCFG201841, GK2250260018, 3072019CF2504, 3072019CF2506, 3072020CFJ2507), the Open Fund of the State Key Laboratory on Integrated Optoelectronics (IOSKL2016KF03), the 111 project to the Harbin Engineering University (B13015), the Science Foundation Ireland under the Centre research program for the MaREI project (SFI/12/RC/2302_P2).

\section{References}

[1] X. Zhou, Z. Liu, Z. Xie, X. Liu, Z. Zheng, High-resolution, large-area, serial fabri cation of 3D polymer brush structures by parallel dip-pen nanodisplacement lithography, Small 8 (2012) 3568-3572.

[2] J. Kokorian, F. Buja, W.M.V. Spengen, In-plane displacement detection with picometer accuracy on a conventional microscope, J. Microelectromech. Syst. 24 (2015) 618-625.

[3] Y. Yu, H. Tam, W. Chung, M.S. Demokan, Fiber Bragg grating sensor for simultaneous measurement of displacement and temperature, Opt. Lett. 25 (2000) 1141-1143.

[4] C. Shen, C. Zhong, Novel temperature-insensitive fiber Bragg grating sensor for displacement measurement, Sens. Actuators, A 170 (2011) 51-54.

[5] P. Kisała, D. Harasim, J. Mroczka, Temperature-insensitive simultaneous rotation and displacement (bending) sensor based on tilted fiber Bragg grating, Opt. Express 24 (2016) 29922-29929.

[6] L. Qi, C.-L. Zhao, Y. Wang, J. Kang, Z. Zhang, S. Jin, Compact micro-displacement sensor with high sensitivity based on a long-period fiber grating with an air-cavity, Opt. Express 21 (2013) 3193-3200.

[7] Q. Wu, Y. Semenova, P. Wang, A.M. Hatta, G. Farrell, Experimental demonstration of a simple displacement sensor based on a bent single-mode-multimode-singlemode fiber structure, Meas. Sci. Technol. 22 (2011) 025203.

[8] K. Tian, G. Farrell, X. Wang, E. Lewis, P. Wang, Highly sensitive displacement sensor based on composite interference established within a balloon-shaped bent multimode fiber structure, Appl. Opt. 57 (2018) 9662-9668.

[9] J. Chen, J. Zhou, Z. Jia, High-sensitivity displacement sensor based on a bent fiber Mach-Zehnder interferometer, IEEE Photonics Technol. Lett. 25 (2013) 2354-2357.

[10] C. Shen, Y. Wang, J. Chu, Y. Lu, Y. Li, X. Dong, Optical fiber axial micro-displacement sensor based on Mach-Zehnder interferometer, Opt. Express 22 (2014) 31984-31992.

[11] M. Bravo, A.M.R. Pinto, M. Lopez-Amo, J. Kobelke, K. Schuster, High precision micro-displacement fiber sensor through a suspended-core Sagnac interferometer, Opt. Lett. 37 (2012) 202-204.

[12] X. Zhou, Q. Yu, Wide-range displacement sensor based on fiber-optic Fabry-Perot interferometer for subnanometer measurement, IEEE Sens. J. 11 (2011) 1602-1606.

[13] X. Liu, Y. Zhao, R. Lv, Q. Wang, High sensitivity balloon-like interferometer for refractive index and temperature measurement, IEEE Photonics Technol. Lett. 28 (2016) 1485-1488.

[14] K. Tian, G. Farrell, E. Lewis, X. Wang, H. Liang, P. Wang, A high sensitivity temperature sensor based on balloon-shaped bent SMF structure with its original polymer coating, Meas. Sci. Technol. 29 (2018) 085104.

[15] C. Trono, F. Baldini, M. Brenci, F. Chiavaioli, M. Mugnaini, Flow cell for strain- and temperature-compensated refractive index measurements by means of cascaded optical fibre long period and Bragg gratings, Meas. Sci. Technol. 22 (2011) 075204.

[16] Q. Yao, H. Meng, W. Wang, H. Xue, R. Xiong, B. Huang, et al., Simultaneous measurement of refractive index and temperature based on a core-offset MachZehnder interferometer combined with a fiber Bragg grating, Sens. Actuators, A 209 (2014) 73-77.

[17] Y. Zhou, W. Zhou, C.C. Chan, W.C. Wong, L.-Y. Shao, J. Cheng, et al., Simultaneous measurement of curvature and temperature based on PCF-based interferometer and fiber Bragg grating, Opt. Commun. 284 (2011) 5669-5672.

[18] R. Zhang, S. Pu, Y. Li, Y. Zhao, Z. Jia, J. Yao, Y. Li, Mach-Zehnder interferometer cascaded with FBG for simultaneous measurement of magnetic field and temperature, IEEE Sens. J. 19 (2019) 4079-4083.

[19] Z. Jia, S. Pu, J. Rao, Y. Zhao, Y. Li, T. Yao, Temperature self-compensative all-fiber magnetic field sensing structure based on no-core fiber cascaded with fiber Bragg gratings, Opt. Lasers Eng. 119 (2019) 26-29.

[20] K. Tian, G. Farrell, W. Yang, X. Wang, E. Lewis, P. Wang, Simultaneous measurement of displacement and temperature based on a balloon-shaped bent SMF 
structure incorporating an LPG, J. Lightwave Technol. 36 (2018) 4960-4966.

[21] D.W. Kim, F. Shen, X. Chen, A. Wang, Simultaneous measurement of refractive index and temperature based on a reflection-mode long-period grating and an intrinsic Fabry-Perot interferometer sensor, Opt. Lett. 30 (2005) 3000-3002.

[22] Y. Zhao, X. Liu, R.-Q. Lv, Q. Wang, Simultaneous measurement of RI and temperature based on the combination of Sagnac loop mirror and balloon-like interferometer, Sens. Actuators, B 243 (2017) 800-805.

[23] D.J. Juan Hu, J.L. Lim, M. Jiang, Y. Wang, F. Luan, P. Ping Shum, et al., Long period grating cascaded to photonic crystal fiber modal interferometer for simultaneous measurement of temperature and refractive index, Opt. Lett. 37 (2012) 2283-2285.
[24] P. Liu, Y. Shi, Simultaneous measurement of refractive index and temperature using cascaded side-coupled photonic crystal nanobeam cavities, Opt. Express 25 (2017) 28398-28406.

[25] P. Chen, X. Shu, K. Sugden, Compact assembly-free vector bend sensor based on allin-fiber-core Mach-Zehnder interferometer, Opt. Lett. 43 (2018) 531-534.

[26] K. Tian, M. Zhang, G. Farrell, R. Wang, E. Lewis, P. Wang, Highly sensitive strain sensor based on composite interference established within S-tapered multimode fiber structure, Opt. Express 26 (2018) 33982-33992.

[27] R.T. Schermer, J.H. Cole, Improved bend loss formula verified for optical fiber by simulation and experiment, IEEE J. Quantum Electron. 43 (2007) 899-909. 